\title{
Association between component-resolved diagnosis of house dust mite and efficacy of allergen immunotherapy in allergic rhinitis patients
}

\author{
Yanran Huang ${ }^{1 \dagger}$, Chengshuo Wang ${ }^{1 \dagger}$, Xi Lin ${ }^{1,2,3}$, Hongfei Lou' ${ }^{1}$, Feifei Cao ${ }^{3}$, Wenhan Li, ${ }^{4,5}$, Yuan Zhang ${ }^{1,2,3^{*}}$
} and Luo Zhang ${ }^{1,2,3^{*}}$ (D)

\begin{abstract}
Data regarding clinical relevance of house dust mite (HDM) components over allergen immunotherapy (AIT) for allergic rhinitis (AR) are lacking. 18 adult AR patients receiving HDM-AIT for 52 weeks were followed up to assess serum levels of slgE and slgG4 to HDM components. The study showed that Der p1, p2, p23, Der f1 and f2, are important sensitizing components of HDM, of which Der p1 appears to be the most clinically relevant allergenic component for effective AIT.
\end{abstract}

Keywords: Allergic rhinitis, Allergen immunotherapy, House dust mite, Component resolved diagnosis

To the editor,

Allergic rhinitis (AR) affects $10-40 \%$ of the global population and exerts huge economic and social burdens [1]. The most important allergen responsible for perennial AR (PAR) in China is HDM, including components Dermatophagoides farina (Der f) and Dermatophagoides pteronyssinus (Der p) [2].

Component resolved diagnostics (CRD) makes it possible for precision medication and individual management, while Der p 1, Der p 2, Der p 23, Der f 1 and Der f 2 are considered to be the main HDM allergens [3-5]. Allergen immunotherapy (AIT), including mainly subcutaneous immunotherapy (SCIT) and sublingual immunotherapy, is the only available etiological treatment for AR. Studies have shown that the levels of SIgE and sIgG4 actually

\footnotetext{
*Correspondence: summer_zhang1211@126.com; dr.luozhang@139.com †Yanran Huang and Chengshuo Wang contributed equally in this paper ${ }^{2}$ Beijing Key Laboratory of Nasal Diseases, Beijing Institute of Otolaryngology, No. 17, HouGou HuTong, Dongcheng District, Beijing 100005, China
}

Full list of author information is available at the end of the article increase during AIT [6, 7]. The levels of specific IgE present an early increase and a late decrease, while the levels of specific IgG4 show a relatively early increase instead [8]. Indeed, both sIgE and sIgG4 have been documented as potential biomarkers for monitoring clinical efficacy of AIT [9]. However, data regarding the clinical relevance of association between specific components of HDM and sIgE and sIgG4 levels during the course of AIT are still lacking. Thus, the aim of this study was to analyze the different HDM components and the profiles of sIgEs and sIgG4s generated against these components over a 1-year course of AIT in patients with HDM-induced AR.

The study was approved by the ethics review board of the Beijing TongRen Hospital (TRECKY2017-06). Study methods are described in Additional file 1 and a schematic diagram is provided in Additional file 1: Figure S1.

A total of 18 adult AR patients, receiving HDM-AIT for 52 weeks were serially followed up to assess serum levels of SIgE and sIgG4 to allergenic HDM components over the 52-week treatment. The demographic and clinical 
characteristics are shown in Additional file 1: Table S1. Clinically, symptoms were significantly improved during the 52-week HDM-AIT (detailed results were shown in Additional file 1).

Additional file 1: Table S2 shows the sensitization patterns of sIgE and sIgG4 to allergenic HDM components at baseline (W0), and indicate that the prevalence of sensitization to the individual components was in the order Der p $2(66.7 \%)=$ Der f $2(66.7 \%)>$ Der p $1(55.6 \%)>$ Der f $1(44.4 \%)>$ Der p 23 (33.3\%); where sensitization to specific allergenic HDM components leading to generation of significant levels of sIgG was in the order Der $\mathrm{f}$ $2(38.9 \%)>$ Der p $1(27.8 \%)=$ Der p23 $(27.8 \%)>$ Der p 2 $(22.2 \%)=$ Der p $3(22.2 \%)$. All participants were found to be negative (EAST class 0 ) with respect to significant generation of sIgE against Der p 3, Der p 5 and Der p 10; and generation of sIgG4 against Der p3, Der p10 and Der p23. Moreover, sIgE against Der p 7 and sIgG4 against Der p 7 and Der $\mathrm{f} 1$ was found to be of little relevance, as they demonstrated intensities below 2 (clinically grade 0 ).
However, $22 \%$ patients $(4 / 18)$ were found to have clinically relevant sIgG4 levels against Der $\mathrm{p} 3$.

The trends of the allergenic HDM components leading to production of sIgE are shown in Fig. 1. During the course of AIT, compared with W0 (baseline), the level of Der p sIgE was significantly elevated at W5 $(25.27 \pm 16.29$ versus $20.13 \pm 15.19, \mathrm{P}=0.022)$, and then decreased at W19 $(15.53 \pm 13.79, \mathrm{P}=0.047)$ before progressively attaining levels comparable with baseline at W52 $(18.93 \pm 16.04$, $\mathrm{P}=0.76$ ). The production of Der $\mathrm{p} 1$ and Der $\mathrm{p} 2$ sIgE followed a trend consistent with Der p sIgE. In contrast, the levels of Der p $23 \mathrm{sIgE}$ were significantly increased from baseline at W2 $(25.61 \pm 37.73$ versus $18.56 \pm 27.86$, $\mathrm{P}=0.027)$, and then remained significantly higher than the baseline throughout the rest of the treatment period (W5, $33.17 \pm 43.64, \mathrm{P}=0.0078 ; \mathrm{W} 7,30.67 \pm 38.31, \mathrm{P}=0.025$; W19, $\quad 28.89 \pm 28.40, \quad \mathrm{P}=0.028 ; \quad \mathrm{W} 35, \quad 30.29 \pm 28.10$, $\mathrm{P}=0.049 ; \mathrm{W} 52,37.11 \pm 25.2, \mathrm{P}=0.0174)$.
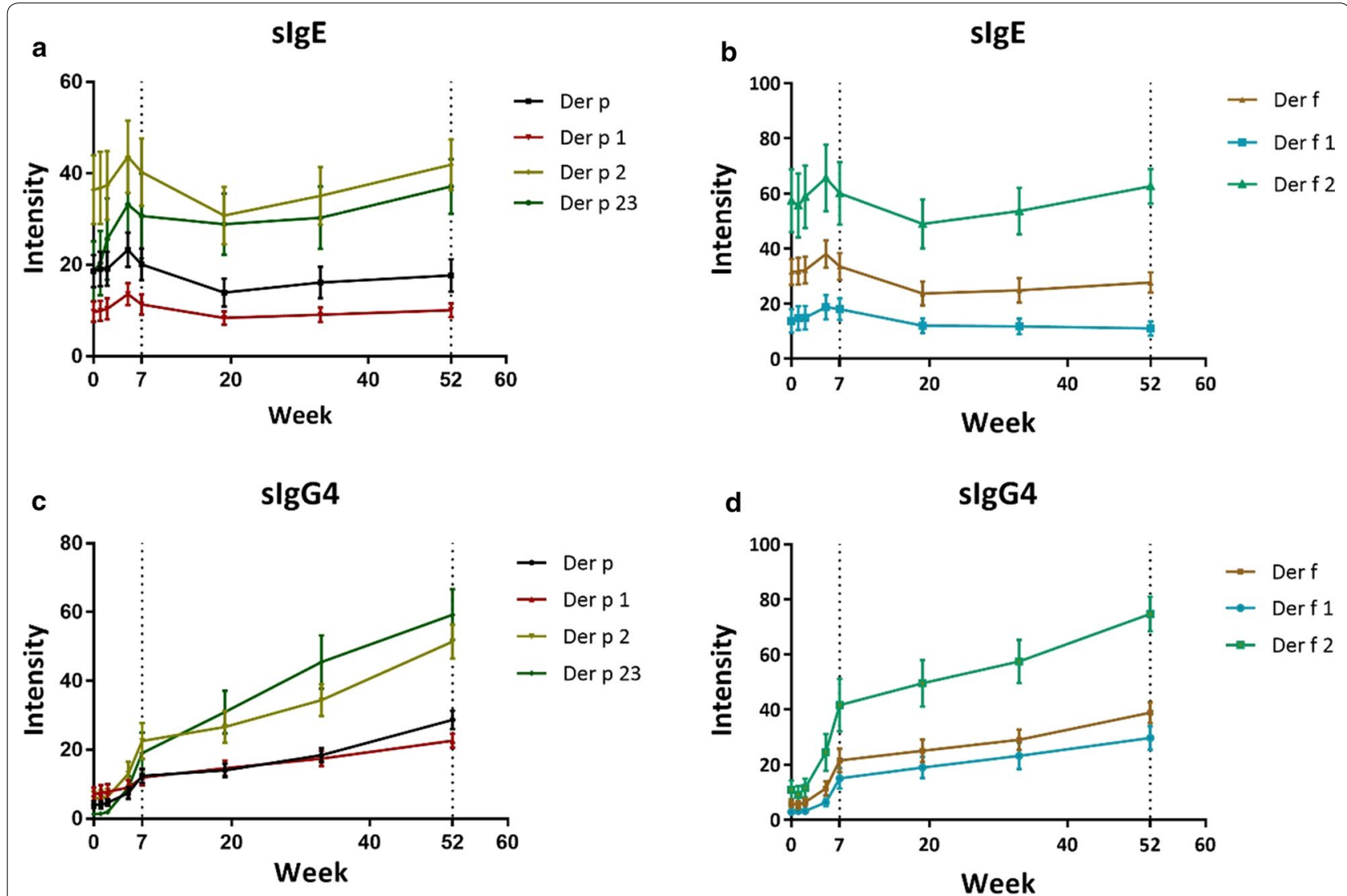

Fig. 1 The profiles of slgE and slgG4 levels generated for allergenic HDM components during AIT. a slgE of Der p and its components; $\mathbf{b}$ slgE of Der $f$ and its components; c slgG4 of Der $p$ and its components; $\mathbf{d}$ slgG4 of Der $f$ and its components. HDM, house dust mite; AIT, allergen immunotherapy 

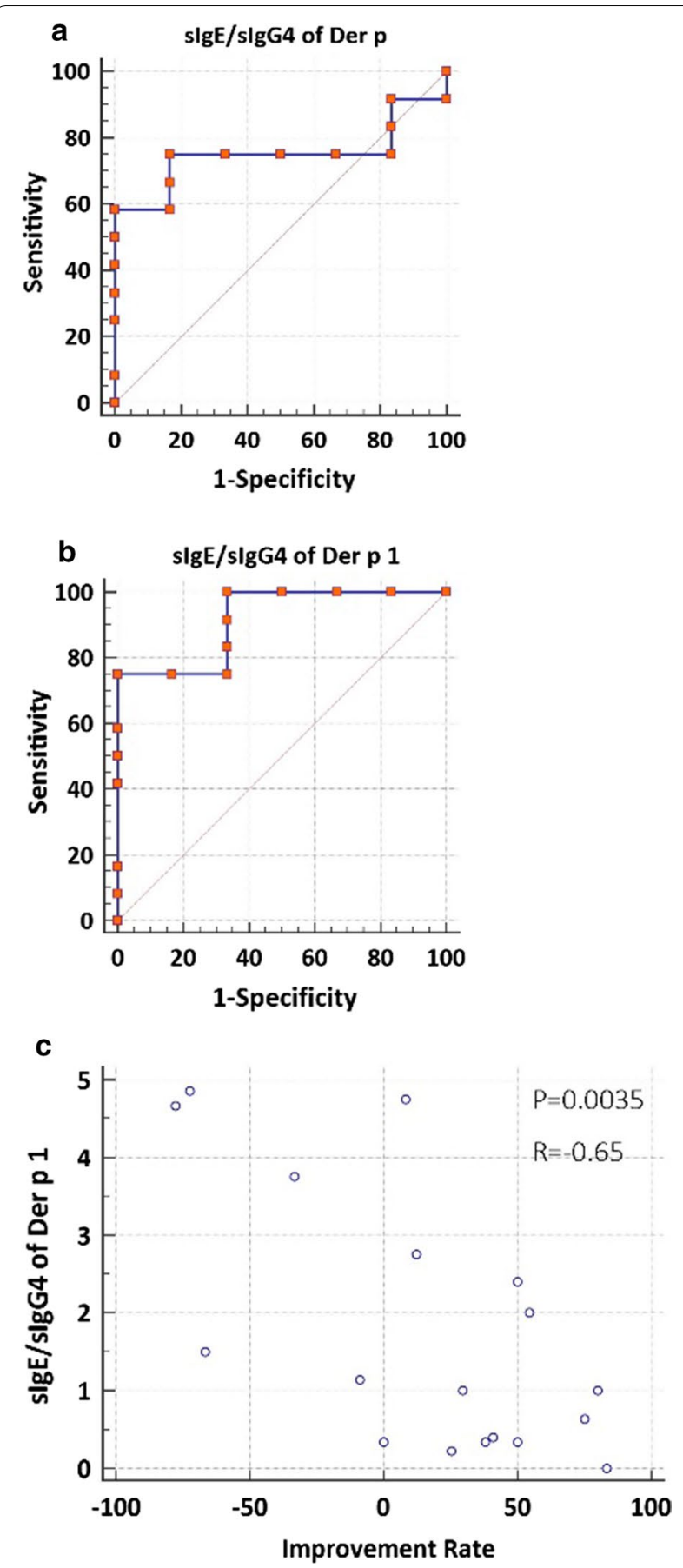

Fig. 2 Receiver operating characteristic (ROC) curve of the baseline slgE/slgG4 ratio of Der $p(\mathbf{a})$ and Der $p 1$ (b) as predictive parameters of clinical responsiveness, and the correlation between baseline slgE/slgG4 ratio for Der $p 1$ and clinical improvement rate for TCS at 52-weeks of AIT (c). AIT, allergen immunotherapy

Similar to Der p sIgE, the level of Der f sIgE was also significantly elevated at W5 compared to baseline level (38.06 \pm 20.77 versus $31.56 \pm 19.94, \mathrm{P}=0.0049)$, and then decreased at W19 $(23.67 \pm 18.56, \mathrm{P}=0.020)$. Likewise, the levels of Der $\mathrm{f} 1$ and Der $\mathrm{f} 2 \mathrm{sIgE}$ significantly increased at W5 (Der $\mathrm{f} 1,18.78 \pm 18.87$ versus $13.78 \pm 18.05, \mathrm{P}<0.0001$; Der f 2, $65.61 \pm 51.3$ versus $57.33 \pm 48.23, \mathrm{P}<0.0001$ ), but remained comparable at the other time-points.

The trends of the specific HDM components leading to production of sIgG4 are shown in Fig. 1. The levels of sIgG4 against Der $\mathrm{p}$, Der $\mathrm{f}$ and their components (Der $\mathrm{p} 1, \mathrm{p} 2, \mathrm{p} 23, \mathrm{f} 1$ and $\mathrm{f} 2$ ) were increased rapidly during the first 7 weeks of treatment and then progressively increased at a slower rate over the remaining course of the 52-weeks treatment.

The clinical efficacy of AIT was defined according the percentage improvement observed in total combined score (TCS) at $52 \mathrm{~W}$ compared with the baseline (W0) (with $\geq 20 \%$ improvement $=$ responder; $<20 \%$ improvement $=$ nonresponder) [10]. 6 out of 18 patients were unresponsive to HDM-SCIT and 12 patients responsive HDM-SCIT. Receiver operating characteristic curve analysis demonstrated that the ratio of sIgE and sIgG4 for Der p and Der p 1 were good predictors for clinical responsiveness, as demonstrated by areas under the curves (AUCs) of 0.75 and 0.92, respectively (Fig. 2a, b). Further, the Der p1 sIgE/sIgG4 was significantly and moderately correlated with the rate of clinical improvement in the patients ( $\mathrm{R}=-0.65, \mathrm{P}=0.0035$; Fig. 2c).

A limitation of the study is that a 52 -week course for AIT is relatively short compared to the traditional 3 -year course for AIT. Secondly, the study population was relatively small.

In summary, this study has provided preliminary information of the allergenic profiles of major HDM components; especially Der p 1, Der p 2, Der p 23, Der $\mathrm{f} 1$ and Der f 2; at baseline and over a course of 52-week AIT in patients with HDM-induced AR. Furthermore, Der $\mathrm{p} 1$ appears to be the most clinically relevant allergenic component for effective AIT, and the ratio of Der p 1-sIgE/Der p 1-sIgG4 levels may be useful as a biomarker for predicting the clinical responses of AIT.

\section{Supplementary information}

Supplementary information accompanies this paper at https://doi. org/10.1186/s13601-019-0305-4.

Additional file 1. Supplemental materials and methods. Demographic and clinical characteristics of the study population at baseline and clinical evaluation. Table S1. Demographics and baseline disease characteristics of participants. Table S2. Baseline levels of slgE and slgG4. Figure S1. Schematic diagram showing the SCIT protocol employed. Figure S2. The effect of HDM-AIT on nasal/conjunctivitis symptoms (A nasal congestion, $B$ rhinorrhea, $C$ nasal itching, D sneezing, E gritty eyes, F watery eyes) scores over a course of 52-weeks. AIT, allergen immunotherapy. Figure S3. The effect of HDM-AIT on DMS (A) and TCS (B) over a course of 52-weeks. DMS, daily medication score; TCS, total combined score; AIT, allergen immunotherapy. 


\begin{abstract}
Abbreviations
AR: allergic rhinitis; AIT: allergen immunotherapy; CRD: component resolved diagnostics; Der f: Dermatophagoides farina; Der p: Dermatophagoides pteronyssinus; HDM: house dust mite; PAR: perennial allergic rhinitis; SCIT: subcutaneous immunotherapy; slgE: specific immunoglobulin E; slgG4: specific immunoglobulin G4; TCS: total combined score; tlgE: total immunoglobulin E; WAO: World Allergy Organization.
\end{abstract}

\section{Acknowledgements}

Not applicable.

\section{Authors' contributions}

HY, WC, ZY and ZL performed statistical analyses. All authors collected the data. All authors read and approved the final manuscript.

\section{Funding}

This work was supported by grants from the program for National Key R \& D Program of China (2016YFC20160905200), the National Natural Science Foundation of China (81570895 and 81420108009), Changjiang scholars and innovative research team (IRT13082), the special fund of capital health development (2011-1017-06, 2011-1017-02), the special fund of sanitation elite reconstruction of Beijing (2009-2-007), Beijing health bureau program for high level talents (2011-3-043), Beijing Municipal Administration of Hospitals' Mission Plan (SML20150203) and Capital citizenry health program (z161100000116062).

\section{Availability of data and materials}

We would like to provide the raw data to support the information presented in this publication.

\section{Ethics approval and consent to participate}

The study was approved by the ethics review board of the Beijing TongRen hospital and Beijing Institute of Otolaryngology, P. R. China (TRECKY2017-06); and written informed consent was obtained from all participants before enrolment into the study.

\section{Consent for publication}

Not applicable.

\section{Competing interests}

The authors declare that they have no competing interests.

\section{Author details}

${ }^{1}$ Department of Otolaryngology Head and Neck Surgery, Beijing TongRen Hospital, Capital Medical University, Beijing 100730, China. ${ }^{2}$ Beijing Key Laboratory of Nasal Diseases, Beijing Institute of Otolaryngology, No. 17, HouGou HuTong, Dongcheng District, Beijing 100005, China. ${ }^{3}$ Department of Allergy, Beijing TongRen Hospital, Capital Medical University, Beijing 100730, China. ${ }^{4}$ EUROIMMUN Medical Diagnostics (China) Co., Ltd, Hangzhou 310013, China.

${ }^{5}$ Oumeng V Medical Laboratory, Hangzhou 310013, China.
Received: 14 November 2019 Accepted: 8 December 2019

Published online: 19 December 2019

\section{References}

1. Brożek JL, Bousquet J, Agache I, Agarwal A, Bachert C, Bosnic-Anticevich $\mathrm{S}$, et al. Allergic Rhinitis and its Impact on Asthma (ARIA) guidelines-2016 revision. J Allergy Clin Immunol. 2017;140:950.

2. Lou H, Ma S, Zhao Y, Cao F, He F, Liu Z, et al. Sensitization patterns and minimum screening panels for aeroallergens in self-reported allergic rhinitis in China. Sci Rep. 2017;7:9286.

3. Zidarn M, Robic M, Krivec A, Silar M, Resch-Marat Y, Vrtala S, et al. Clinical and immunological differences between asymptomatic HDM-sensitized and HDM-allergic rhinitis patients. Clin Exp Allergy. 2019;49(6):808-18.

4. Matricardi PM, Dramburg S, Potapova E, Skevaki C, Renz H. Molecular diagnosis for allergen immunotherapy. J Allergy Clin Immunol. 2019;143:831-43.

5. Matos Semedo F, Dorofeeva Y, Pires AP, Tomaz E, Taborda Barata L, Inacio F, et al. Der p 23-clinical relevance of molecular monosensitisation in House Dust Mite allergy. J Investig Allergol Clin Immunol. 2019;29(4):314-6.

6. Zeng G, Zheng P, Luo W, Huang H, Wei N, Sun B. Longitudinal profiles of serum specific lgE and lgG4 to Dermatophagoides pteronyssinus allergen and its major components during allergen immunotherapy in a cohort of southern Chinese children. Mol Immunol. 2016;74:1-9.

7. Suarez-Fueyo A, Ramos T, Galan A, Jimeno L, Wurtzen PA, Marin A, et al. Grass tablet sublingual immunotherapy downregulates the $\mathrm{TH} 2$ cytokine response followed by regulatory T-cell generation. J Allergy Clin Immunol. 2014:133:130-8.

8. Fujita H, Soyka MB, Akdis M, Akdis CA. Mechanisms of allergen-specific immunotherapy. Clin Transl Allergy. 2012;2:2

9. Shamji MH, Kappen JH, Akdis M, Jensen-Jarolim E, Knol EF, Kleine-Tebbe J, et al. Biomarkers for monitoring clinical efficacy of allergen immunotherapy for allergic rhinoconjunctivitis and allergic asthma: an EAACI Position Paper. Allergy. 2017;72:1156-73.

10. Canonica GW, Baena-Cagnani CE, Bousquet J, Bousquet PJ, Lockey RF, Malling $\mathrm{HJ}$, et al. Recommendations for standardization of clinical trials with Allergen Specific Immunotherapy for respiratory allergy. A statement of a World Allergy Organization (WAO) taskforce. Allergy. 2007;62(3):317-24.

\section{Publisher's Note}

Springer Nature remains neutral with regard to jurisdictional claims in published maps and institutional affiliations.
Ready to submit your research? Choose BMC and benefit from:

- fast, convenient online submission

- thorough peer review by experienced researchers in your field

- rapid publication on acceptance

- support for research data, including large and complex data types

- gold Open Access which fosters wider collaboration and increased citations

- maximum visibility for your research: over 100M website views per year

At BMC, research is always in progress.

Learn more biomedcentral.com/submissions 